Conclusion This survey demonstrates hospices to be interested and active in facilitating social support. It is possible that increasingly diverse approaches used in practice may improve issues regarding access to and awareness of hospice care. More work is needed to share best practice, document outcomes, and consider cost-effectiveness.

\section{0-22 HOLISTIC (HOSPICE-LED INNOVATIONS STUDY TO IMPROVE CARE)}

${ }^{1}$ Jonathan Ellis, ${ }^{1}$ Ros Taylor, ${ }^{2}$ Steven Walker, ${ }^{2}$ Claire Hawksworth, ${ }^{3}$ Giles Colclough. ${ }^{1}$ Hospice UK, London, UK; ${ }^{2}$ St Giles Medical, London, UK; ${ }^{3}$ McKinsey and Company, London, UK

\subsection{6/bmispcare-2018-hospiceabs.22}

Background A current concern is the number of people dying in hospital who have no medical need, or wish, to be there (Marie Curie Cancer Care, 2012). 72\% of people would prefer to die at home (ComRes, 2014), yet just 25\% do so, with $50 \%$ dying in hospital (Gomes, Calanzani, Higginson, 2011). Instinctively, hospice-led initiatives play an important role in minimising inappropriate hospital usage at the end of life, but there is a lack of robust data.

Aim To establish the impact of different hospice led innovations on a) reducing the number of hospital bed days during the last 90 days of life, b) place of death and other secondary outcome measures: the number of emergency and inpatient admissions and discharges to a hospice in the last 90 days of life.

Method Mixed methods study with a quantitative quasi-experimental longitudinal design employing a 'difference of difference' analysis of HES data to assess the impact on hospital utilisation in the last 90 days of life. Any encountered differences are compared to control cohorts. Stakeholders were qualitatively interviewed through open-ended, semi-structured and structured interviews followed by narrative, framework and content analysis respectively.

Results Quantitative: ongoing, however, we anticipate data showing a reduction in the number of hospital beds days, in the last 90 days of life, within the locality of the intervention hospice. Qualitative: interviewed 188 people, including 24 patients and carers, across 27 interventions at 25 sites providing 31 recurrent topics of which the five most relatively frequent were the process of development, collaboration, the intervention group, staff roles and professional culture.

Conclusions Qualitative evaluation of these innovations shows benefit to the patient experience and factors critical to success and replicability. Quantitative data will show the impact on NHS resources, and together the findings will enable better evidence-based commissioning, supporting service redesign at a local level. Final report due Sept. 2018.

\section{Parallel session 7: Engaging patients and the wider public}

\section{0-23 PATIENT AND PUBLIC INVOLVEMENT (PPI) CONTRIBUTIONS TO PALLIATIVE CARE RESEARCH}

Clare Gardiner. The University of Sheffield, Sheffield, UK

10.1136/bmispcare-2018-hospiceabs.23
Background Patient and public involvement (PPI) is an established component of high quality palliative care research. The way patients and public are involved in research differs between and across research studies, however the range of contributions and different models of working are not well documented in palliative care.

Aim The aim is to document the range and scope of activities of a service user advisory panel - the Palliative Care Studies Advisory Group (PCSAG), established at The University of Sheffield in 2009.

Methods A narrative summary of PPI activities, documenting the range and scope of contributions to research over the nine years since the PCSAG was established.

Results Since they were established the group have contributed to 34 research projects and funding applications in the area of palliative and end of life care. These have ranged from substantial multi-million pound international research studies to $\mathrm{PhD}$ studies and student projects. In the main, researchers attended facilitated group discussions to request advice on aspects of their research projects, however, other models of working have emerged in more recent years. These include group members sitting on project steering committees as coapplicants, contributing to data analysis and presenting at conferences, and developing on-line methods of providing PPI input.

Discussion The Palliative Care Studies Advisory Group have made a substantial contribution to palliative and end of life care research. The scope and depth of PPI involvement has increased over the years, as researchers and service users have gained confidence in working together. Models of co-working which involve service users as co-applicants have more recently become commonplace as the PPI movement has become more embedded in research. However, little guidance is available to support researchers working with service users in palliative and end of life care, and further research could address this gap.

\section{0-24 END OF LIFE CARE AND DECISION MAKING: PUBLIC KNOWLEDGE, NEEDS AND PREFERENCES IN WALES}

Ishrat Islam, Anthony Byrne, Annmarie Nelson. Cardiff University, Cardiff, UK

\subsection{6/bmispcare-2018-hospiceabs.24}

Introduction A prudent approach to provide quality end of life care (EoLC) advocates for active participations of the patients in designing the service (Wynne, n.d.). Patients and carers demand for improvements in decision-making at the EoLC (Sinuff et al, 2015). But there is a lack of evidence on understanding of people's attitudes towards EoLC and shared decision-making (Hajizadeh, Uhler, Herman, et al., 2016).

Aim To increase understanding of people's views, feelings and preferences around EoLC and decision making.

Method An online survey was conducted using an electronic survey tool. Descriptive statistics and thematic analysis were used to perform data analysis.

Results 2210 people (Age: Mean \pm SD; $55 \pm 14$ years) participated in the survey, $43 \%$ of those were members of the public, 29\% were patients/carers, and $23 \%$ were health professionals. $49 \%$ of respondents thought that available EoLC and palliative care was inadequate and only 31\% believed that people could access these facilities. More than $60 \%$ 